\title{
Refractive Index Determination of Nanoparticles in Suspension Using Nanoparticle Tracking Analysis
}

\author{
Edwin van der Pol, ${ }^{*}, \dagger$ Frank A. W. Coumans, ${ }^{\dagger, \ddagger}$ Auguste Sturk, ${ }^{\ddagger}$ Rienk Nieuwland, ${ }^{\ddagger}$ \\ and Ton G. van Leeuwen ${ }^{\dagger}$ \\ ${ }^{\dagger}$ Biomedical Engineering and Physics, ${ }^{\ddagger}$ Laboratory of Experimental Clinical Chemistry, Academic Medical Center, University of \\ Amsterdam, Amsterdam, The Netherlands
}

Supporting Information
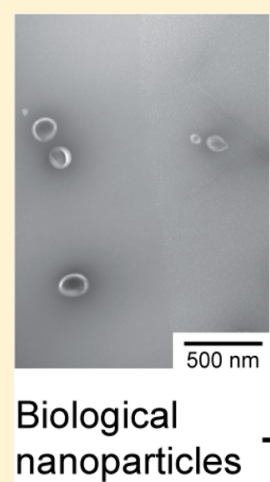

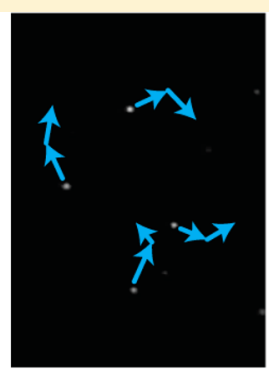

Single particle tracking

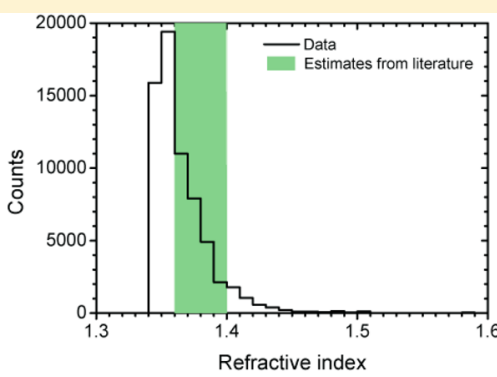

Refractive index determination

ABSTRACT: The refractive index (RI) dictates interaction between light and nanoparticles and therefore is important to health, environmental, and materials sciences. Using nanoparticle tracking analysis, we have determined the RI of heterogeneous particles $<500 \mathrm{~nm}$ in suspension. We demonstrate feasibility of distinguishing silica and polystyrene beads based on their RI. The hitherto unknown RI of extracellular vesicles from human urine was determined at 1.37 (mean). This method enables differentiation of single nanoparticles based on their RI.

KEYWORDS: Drug delivery, exosomes, extracellular vesicles, microparticles, nanomedicine, nanoparticles

$\mathrm{T}$ he refractive index (RI) of nanoparticles is an indispensable property in a wide range of applications and studies but is difficult to measure. ${ }^{1}$ The RI depends on the wavelength and is defined as the ratio of speed of light in vacuum to speed of light in the material. It relates light scattering to the size, shape, and chemical composition of a nanoparticle ${ }^{2}$ and it defines the magnitude of the optical force that an electromagnetic field exerts on nanoparticles. ${ }^{3,4}$ Because the RI is related to the chemical composition of the particle as well, determination of the RI can also be utilized to differentiate between different components of samples. For example, in atmospheric particulate matter, pollen $(\mathrm{RI} \approx 1.53)^{5}$ could be distinguished from cement dust $(\mathrm{RI} \approx 1.70)^{6}$ and fly ash $(\mathrm{RI}=$ $1.55-1.60){ }^{7}$ In clinical samples, it may be possible to distinguish vesicles from similar-sized lipoproteins $(\mathrm{RI}=$ $1.45-1.60),{ }^{8}$ which are abundantly present in blood. ${ }^{8}$ Potential differentiation of other nanoparticles by RI include soda lime, borosilicate, calcium carbonate, aluminum oxide, aluminum silicate, diamond, gold, nickel, poly(methyl methacrylate) (PMMA), polytetrafluoroethylene (PTFE), bacteria, viruses, and yeast. Finally, the transfer of the optical properties of bulk to particle is not always straightforward. For example, for gold nanoparticles the choice of dielectric function is still not fully clear, resulting in differences in the prediction of the scattering properties of gold nanorods. ${ }^{9}$ Thus, the RI dictates the interaction between light and nanoparticles in diverse applications spanning environmental science (e.g., the effect of aerosols on climate $\mathrm{c}^{10,11}$ or the carbon content of plankton ${ }^{12}$ ), health (drug delivery, ${ }^{13-15}$ nanotherapy, ${ }^{16}$ imaging contrast, ${ }^{17}$ or photodynamic therapy $\left.{ }^{18,19}\right)$, and materials science (nanoparticles in paint ${ }^{20,21}$ or solar cells ${ }^{22}$ ).

Table 1 shows the capabilities of current techniques for determining the RI of particles. For polydisperse particles with homogeneous RI, the most applied technique is RI matching the medium to the particles. ${ }^{23,24}$ However, RI matching cannot be applied to particles of biological origin due to osmotic effects. Moreover, a sample with heterogeneous RI analyzed with a technique suitable only for homogeneous RI may result in artifacts. For monodisperse particles of known size and concentration, the RI can be determined by measuring the optical extinction coefficient of multiple particles simultaneously. ${ }^{11,25,26}$ Sorting on size may allow these techniques to determine the RI of polydisperse particles. However, size-based fractionation of particles $<500 \mathrm{~nm}$ is difficult and may introduce artifacts. ${ }^{27}$ For samples with heterogeneous RI and unknown

Received: July 1, 2014

Published: September 26, 2014 
Table 1. Capabilities and Requirements of Methods to Determine the Refractive Index (RI) of Particles ${ }^{a}$

\begin{tabular}{|c|c|c|c|c|c|c|c|}
\hline method & references & $\begin{array}{l}\text { in } \\
\text { suspension }\end{array}$ & $\begin{array}{l}\text { diameter } \\
(\mu \mathrm{m})\end{array}$ & $\begin{array}{l}\text { single } \\
\text { particles }\end{array}$ & $\begin{array}{l}\text { heterogeneous } \\
\text { diameter }\end{array}$ & $\begin{array}{l}\text { heterogeneous } \\
\mathrm{RI}^{b}\end{array}$ & requirements \\
\hline RI matching & $23,24,59$ & + & all & - & + & - & altering the medium \\
\hline \multirow[t]{2}{*}{ optical extinction coefficient } & 25 & + & all & - & - & - & $\begin{array}{l}\text { particle concentration and } \\
\text { diameter }\end{array}$ \\
\hline & 11,26 & - & all & - & - & - & $\begin{array}{l}\text { particle concentration and } \\
\text { diameter }\end{array}$ \\
\hline \multirow{3}{*}{$\begin{array}{l}\text { angle/wavelength resolved scattering, } \\
\text { diffraction, optical force }\end{array}$} & $28-30$ & - & $>3.0$ & + & + & + & \\
\hline & $31-33$ & + & $>0.5$ & + & + & + & \\
\hline & $1,34,35$ & + & $>1.0$ & + & + & + & \\
\hline
\end{tabular}

${ }^{a}$ A method that is incapable or capable of providing information on the RI of single particles, particles with a heterogeneous size or RI distribution, or particles in suspension is indicated by - and + , respectively. ${ }^{b}+$ Heterogeneous and homogeneous possible; - only homogeneous possible.

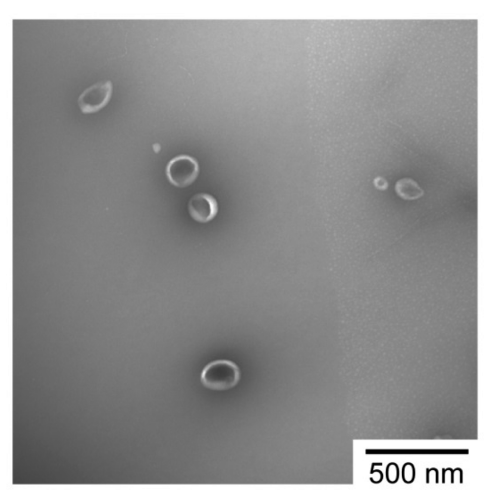

(A)

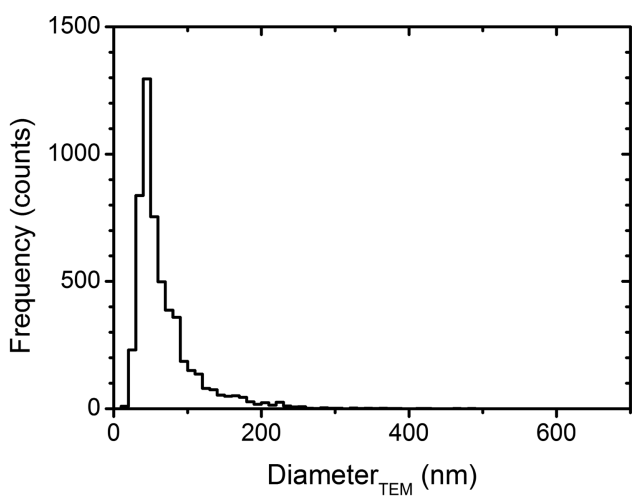

(B)

Figure 1. Size and morphology of urinary vesicles by TEM. (A) TEM image of urinary vesicles. The vesicles have a characteristic cup-shaped morphology. (B) Particle size distribution of 2000 vesicles determined from 25 TEM images. The distribution ranges from 15 to $485 \mathrm{~nm}$ and has a single peak at $45 \mathrm{~nm}$.

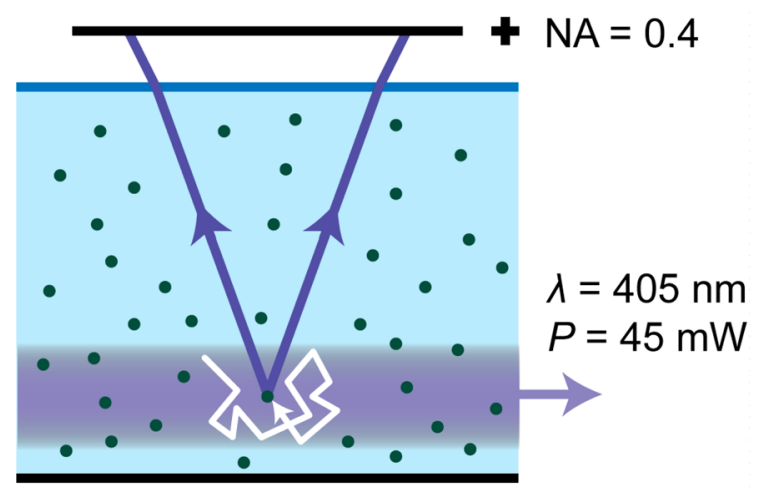

(A)

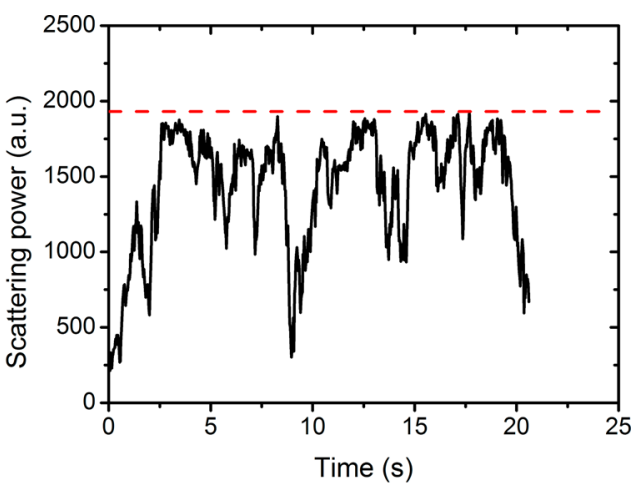

(B)

Figure 2. Detection of light scattered by particles undergoing Brownian motion. (A) Schematic representation of the NTA setup. A laser beam (purple) with a wavelength $(\lambda)$ of $405 \mathrm{~nm}$ and a power $(P)$ of $45 \mathrm{~mW}$ illuminates particles (spheres) in suspension. The particles are undergoing Brownian motion, which is the random motion (white arrow) resulting from collisions with molecules in the suspension. Light scattered by a particle is collected by a microscope objective with a numerical aperture (NA) of 0.4. (B) Scattering power versus time of a $203 \mathrm{~nm}$ polystyrene bead (solid) and the maximum scattering power (dashed). Because of Brownian motion the particle moves through the focal plane and the laser beam, causing the scattering power to fluctuate.

size distribution, the RI can be derived from single particles by measuring angle or wavelength resolved scattering, Fraunhofer diffraction, or the stiffness of an optical trap. ${ }^{1,28-35}$ These techniques, however, have only been applied to particles $>500$ $\mathrm{nm}$. Thus, currently no method is capable of determining RI of single nanoparticles $(<500 \mathrm{~nm})$ in suspension.

Consequently, the RI of extracellular vesicles $<500 \mathrm{~nm}$, such as exosomes, is unknown. Extracellular vesicles are biological nanoparticles that are released by cells to transport waste and exchange intercellular messages, such as DNA, RNA, or surface receptors. ${ }^{36}$ Body fluids, but also seawater, contain typically $10^{5}$ to $10^{10}$ of these vesicles per $\mathrm{mL} .^{37-40}$ Because most vesicles have specialized functions and contribute to homeostatic processes, clinical applications of vesicles are in development. ${ }^{36}$ Figure 1A shows a transmission electron microscopy (TEM) image of vesicles from human urine (see Supporting Information). Urine contains a relatively high concentration of vesicles with low contamination of similar-sized non-cell- 
derived particles. ${ }^{41}$ Figure $1 \mathrm{~B}$ shows the particle size distribution (PSD) of urinary vesicles based on TEM data. The PSD ranges from 25 to $485 \mathrm{~nm}$ with a mode diameter of $45 \mathrm{~nm}$. The small size and large heterogeneity of vesicles are characteristic of biological fluids and hamper their detection. ${ }^{39}$ Please note that other biological fluids, such as blood, contain many components $<1 \mu \mathrm{m}$ with different $\mathrm{RI}$, including protein aggregates $(\mathrm{RI}=1.59-1.64),{ }^{8,42}$ lipoproteins $(\mathrm{RI}=1.45-$ $1.60),{ }^{8}$ and viruses $(\mathrm{RI}=1.52-1.57) .{ }^{43,44}$

Currently, various optical techniques, such as flow cytometry, nanoparticle tracking analysis (NTA), and Raman microspectroscopy, are employed and improved to study vesicles in suspension. ${ }^{37,41,45,46}$ An essential property in these studies is the RI of vesicles. For example, RI determines the smallest detectable vesicle in NTA and flow cytometry, RI determines the smallest vesicle size that can be trapped with Raman microspectroscopy, and RI determines the relationship between size and scatter in flow cytometry. ${ }^{39}$ In flow cytometry, the RI of vesicles has (accidentally) been assumed to be similar to polystyrene beads. This resulted in a gating strategy that selected vesicles with diameters from 800 to $2400 \mathrm{~nm}$, rather than the intended $500-900 \mathrm{~nm}^{45}$ The aim of this work is to develop a method to determine the RI of single nanoparticles in suspension and, as a proof of principle, apply the method to estimate the RI of extracellular vesicles.

We have determined the RI by independently measuring diameter and light scattering power of individual particles with NTA and solving the inverse scattering problem with Mie theory. Figure 2A schematically depicts the operating principle of the NTA. We visualized scattered light from particles illuminated by a $45 \mathrm{~mW} 405 \mathrm{~nm}$ laser by a dark-field microscope (NS500, Nanosight, U.K.). Because of Brownian motion, each particle moved randomly through the suspension. We used the trajectory of each particle in the lateral direction relative to the microscope objective to determine the diffusion coefficient, which we related to the particle diameter via the Stokes-Einstein equation..$^{37,38,47}$ Because the detected scattering power depends on the axial position of a particle, which changed due to Brownian motion, the detected scattering power fluctuated. Figure $2 \mathrm{~B}$ shows a typical measurement of the scattering power versus time for a polystyrene bead with a diameter of $203 \mathrm{~nm}$. Because we focused the objective onto the optical axis of the laser beam, the maximum scattering power was measured when the particle was in focus. To derive the RI, we described the measured (maximum) scattering power $P$ from particles in focus by the theoretical scattering cross section $\sigma_{\text {Mie }}$ from Mie theory ${ }^{2}$ using the measured particle diameter as input to the calculation. Mie theory, extensively described by Bohren and Huffman, ${ }^{2}$ provides an analytical solution of Maxwell's equations and describes light scattering of spheres of all size parameters. However, Mie theory does not reduce these variables to a single equation, since the solution to Maxwell's equations are an infinite series expansions of the electromagnetic fields. We use the Matlab Mie scripts of Mätzler ${ }^{48}$ to calculate the infinite series and obtain the amplitude scattering matrix elements, which describe the relation between the incident and scattered field amplitudes of a sphere. Our model incorporates particle diameter and RI, RI of the medium, and wavelength, polarization, and collection angles of the microscope (see Supporting Information).

To calibrate the NTA Instruments, we measured the scattering power of polystyrene beads $P_{\mathrm{PS}}$ of known size and calculated the scattering cross section of polystyrene spheres $\sigma_{\text {Mie. }}^{\mathrm{PS}}$ The RI of bulk polystyrene is 1.633 at $405 \mathrm{~nm}^{49} \mathrm{We}$ measured $P_{\mathrm{PS}}$ for monodisperse populations of beads (Nanosphere, Thermo Fisher, MA) with a mean diameter of 46, 102, 203,400 , and $596 \mathrm{~nm}$ and a concentration of $10^{8}$ beads $\cdot \mathrm{mL}^{-1}$. For each diameter, five videos of $30 \mathrm{~s}$ were captured with NTA v2.3.0.17 software (Nanosight) and at least 100 particles were tracked. Because the scattering power of the beads differs more than 3 orders of magnitude, each sample required different camera settings (see Supporting Information) to prevent pixel saturation. The videos contain 8 -bit images of 640 by 480 pixels, which were processed with scripts by Blair and Dufresne $^{50}$ in Matlab (v7.13.0.564) to track the particles (see Supporting Information). From the trajectory of each particle, we calculated the mean square displacement and diffusion coefficient and related it to particle diameter via the StokesEinstein equation. Furthermore, the script determined the maximum scattering power of each particle within its trajectory and corrected for the applied shutter time and camera gain. An increase in minimum tracklength increases the precision of the measured diameter and scattering power but also reduces the number of analyzed particles. After the analysis described in the Supporting Information, we required a minimum tracklength of 30 frames. We performed all measurements at $22.0{ }^{\circ} \mathrm{C}$ and assumed a medium viscosity of $0.95 \mathrm{cP}$. To take into account the illumination irradiance and transmission efficiency, the median of $P_{\mathrm{PS}}$ was scaled onto $\sigma_{\mathrm{Mie}}^{\mathrm{PS}}$ by a least-square fit. The resulting scaling factor is 0.067 , which is a property of the instrument that we will use throughout this letter to scale $P$ to $\sigma$. Figure 3 shows $P_{\mathrm{PS}}$ and $\sigma_{\mathrm{Mie}}^{\mathrm{PS}}$ versus particle diameter. The data and theory show good agreement with a coefficient of determination $R^{2}=0.997$.

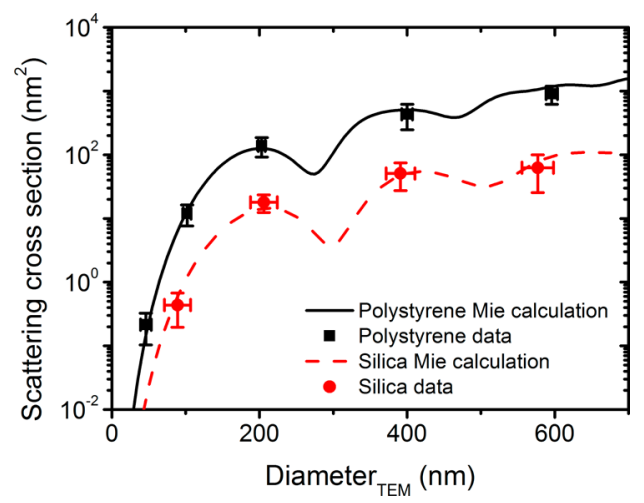

Figure 3. Measured (symbols) and calculated (lines) scattering cross section versus diameter for polystyrene beads (black) and silica beads (red). The scattering cross section increases with increasing particle diameter and refractive index. The particle diameters are determined by TEM. Error bars indicate one standard deviation of the mean.

To validate our approach, we have measured the scattering cross section of monodisperse populations of silica beads (Kisker Biotech, Germany) with a diameter of 89, 206, 391, and $577 \mathrm{~nm}$ and a concentration of $10^{8}$ beads $\cdot \mathrm{mL}^{-1}$, as shown in Figure 3. Since the $\mathrm{RI}$ of silica beads $\left(\mathrm{RI}_{\mathrm{Si}}\right)$ is not exactly known, we performed a least square regression to fit the scattering cross section of silica beads $\sigma_{\text {Mie }}^{\text {Si }}$ to the data. We found a $\mathrm{R}_{\mathrm{Si}}$ of 1.432 , which is in between 1.43 and 1.45 of previous estimates, ${ }^{1,51}$ confirming that NTA can be used to determine the RI of nanoparticles. 
To further validate the method, we determined the PSD and RI distribution of a mixture of $203 \mathrm{~nm}$ polystyrene beads and $206 \mathrm{~nm}$ silica beads with a concentration of $10^{8}$ beads $\cdot \mathrm{mL}^{-1}$ for both populations. We captured 20 videos of $30 \mathrm{~s}$ to track at least 1000 particles. Data were processed as described above. Figure 4A shows measured $\sigma$ versus bead diameter. Each dot represents a single particle, and two populations of beads are clearly discernible. As a reference, the gray lines show $\sigma_{\mathrm{Mie}}$ for seven RIs between 1.35 and 1.65. Figure 4B shows the bead
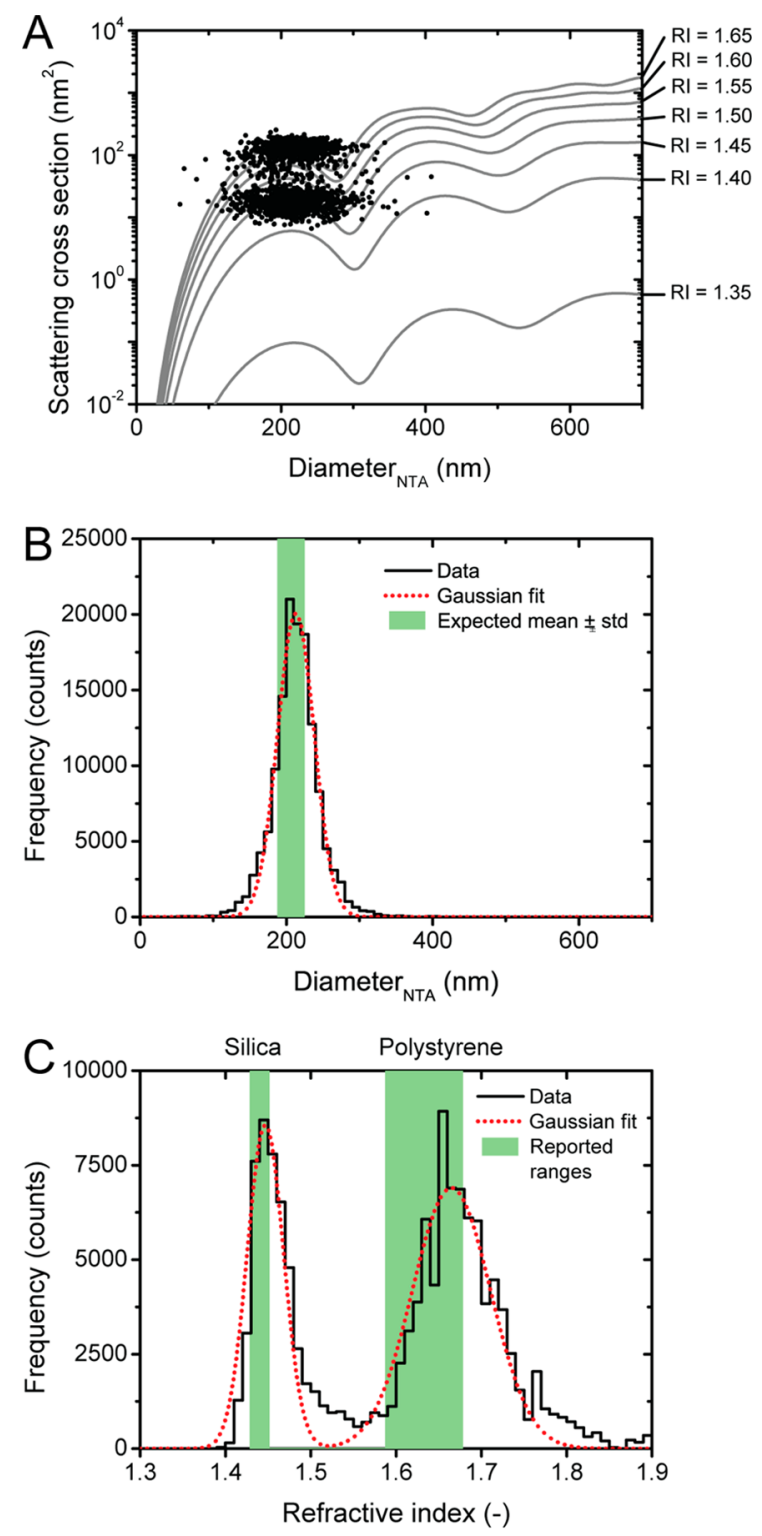

Figure 4. Size and refractive index (RI) determination of a mixture of $203 \mathrm{~nm}$ polystyrene beads and $206 \mathrm{~nm}$ silica beads in water by nanoparticle tracking analysis (NTA). (A) Scattering cross section versus diameter calculated by Mie theory (lines) and measured for the bead mixture (dots). (B) Particle size distribution of the bead mixture (solid line) fitted by a Gaussian function (dotted line; offset $f_{0}=0$, mean $\mu_{0}=213 \mathrm{~nm}$, standard deviation $\mathrm{SD}_{0}=25 \mathrm{~nm}$, area $A_{0}=1.26 \times$ $\left.10^{6}\right)$. The vertical green bar indicates $\mu \pm \mathrm{SD}$ of the $206 \mathrm{~nm}$ silica beads measured by TEM. (C) RI distribution of the bead mixture (solid line) fitted by a sum of two Gaussian functions (dotted line; $f_{1}=$ $0, \mu_{1}=1.447, \mathrm{SD}_{1}=0.021, A_{1}=458, \mu_{2}=1.665, \mathrm{SD}_{2}=0.046, A_{2}=$ $793)$. The vertical green bars indicate the range of reported RIs from literature. mixture PSD obtained by NTA fitted by a Gaussian distribution, which resulted in a size of $213 \pm 25 \mathrm{~nm}$ (mean \pm standard deviation). As a reference, the vertical bar shows the diameter of the silica beads determined by transmission electron microscopy (TEM), which is $206 \pm 18 \mathrm{~nm}$. We attribute the overestimation of the mean diameter to the uncertainty in the measured diffusion coefficient and to the difference between the hydrodynamic diameter measured by NTA and the physical diameter measured by TEM.

Figure 4C shows the RI distribution of the bead mixture and a fit of two Gaussian distributions. We could clearly distinguish silica beads from similar-sized polystyrene beads. We obtained an $\mathrm{RI}_{\mathrm{Si}}$ of $1.447 \pm 0.021$ (mean \pm standard deviation), which is close to 1.432 as derived from Figure 3 and in between 1.43 and 1.45 of previous estimates. ${ }^{1,51}$ For the RI of polystyrene beads $\left(\mathrm{RI}_{\mathrm{PS}}\right)$ we obtained $1.665 \pm 0.046$, which is between 1.59 and 1.68 of previous estimates. ${ }^{11,49}$ Previous estimates with other techniques resulted in standard deviations of $\mathrm{RI}_{\mathrm{PS}}$ between 0.011 and $0.027,,^{1,11,26}$ which is lower than our result. However, those techniques could not detect single particles $<500 \mathrm{~nm}$, and could not detect heterogeneous RI. The precision of $\mathrm{RI}_{\mathrm{PS}}$ measurements with NTA is approximately 2 -fold larger than literature values. We expect that the precision of RI will be reduced for particles with a lower RI than polystyrene, as $\sigma$ becomes more dependent on the RI for such particles. This may partly explain why the standard deviation of $\mathrm{RI}_{\mathrm{Si}}$ was 0.021 . Moreover, because the standard deviation of the diameter scales with $1 /$ tracklength $^{1 / 2}, 52$ increasing the tracklength will reduce the standard deviation of the diameter, $\sigma$ and RI. Technical modifications required to increase the tracklength without reducing the number of particles tracked are feasible but require extensive modifications to the hardware. $^{53}$

As a proof of principle, we applied NTA for the determination of the RI of urinary vesicles from a healthy male individual. After collection, urine was centrifuged twice $\left(50 \mathrm{~mL}, 4^{\circ} \mathrm{C}, 10 \mathrm{~min}, 180 \mathrm{~g}\right.$; and $20 \mathrm{~min}, 1550 \mathrm{~g}$ ) to remove cells and diluted 100-fold in $50 \mathrm{~nm}$ filtered (Nucleopore, GE Healthcare, IL) phosphate-buffered saline (PBS). Figure 5A shows $\sigma$ versus the diameter of urinary vesicles measured by NTA. We captured 20 videos of $30 \mathrm{~s}$ to track at least 1000 vesicles. Because the scattering power of the vesicles differs more than 3 orders of magnitude, we used three different camera settings: gain 100, 350, and 470. The gains were selected such that the range of detectable scattering cross sections overlapped. Data processing was performed as described above. The gray lines again show the relationship between $\sigma$ and the diameter for seven RIs between 1.35 and 1.65 , taking into account that the RI of PBS is 0.002 higher than the RI of water. ${ }^{54}$ Figure $5 \mathrm{~B}$ shows the measured PSD of urinary vesicles. The PSD ranges from 45 to $865 \mathrm{~nm}$ with a mode diameter of $115 \mathrm{~nm}$. Similar to TEM, the right-hand side of the PSD shows a decreasing concentration with increasing diameter, but vesicles smaller than $\sim 100 \mathrm{~nm}$ are below the detection limit for the settings used. Figure 5C shows the measured RI distribution of urinary vesicles with a mean RI of 1.37. The RI of urinary vesicles is lower compared to a previous estimate of plasma vesicles $>500 \mathrm{~nm}$, which have an RI distribution ranging from 1.34 to 1.50 with a peak at $1.40 .^{31}$ However, in contrast to urine, plasma of nonfasting subjects contains chylomicrons, ${ }^{37}$ which are lipoprotein particles with an RI between 1.45 and $1.60 .^{8}$ In addition, plasma vesicles may differ in composition from urinary vesicles. Moreover, our 

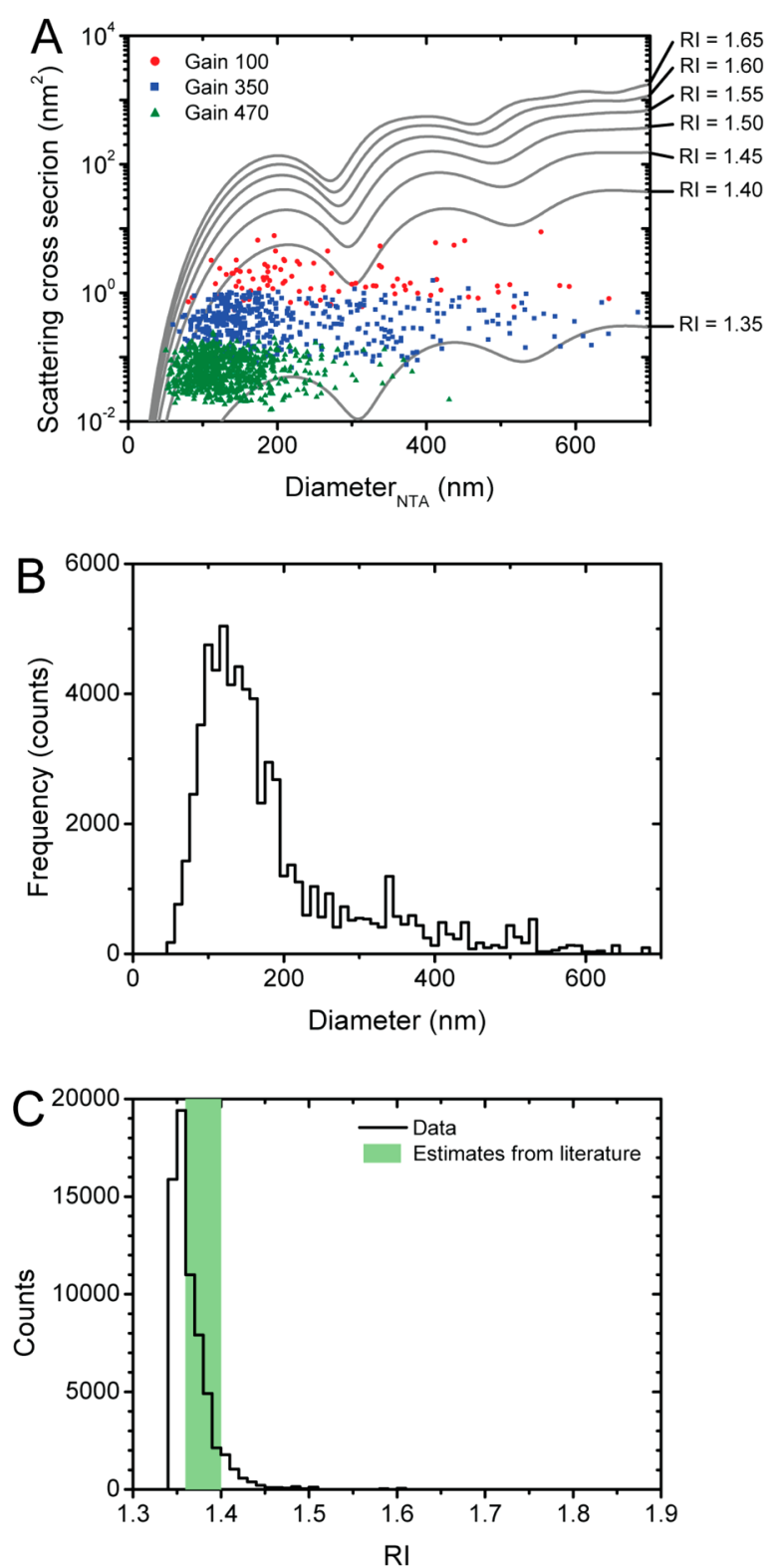

Figure 5. Size and RI determination of urinary vesicles in phosphatebuffered saline by NTA. (A) Scattering cross section versus diameter calculated by Mie theory (lines) and measured for urinary vesicles with camera gain 100 (red circles), 350 (blue squares), and 470 (green triangles). (B) Particle size distribution (black line) of urinary vesicles. (C) RI distribution of urinary vesicles (black) and the range of estimates from literature (vertical green bar).

estimated RI of urinary vesicles falls within the range of estimates $^{41,45,55}$ based on the RI of cells. ${ }^{56-58}$

The true RI of vesicles may differ from our estimate for four reasons. First, the scaling factor that relates scattering power to $\sigma$ is obtained with polystyrene beads for which the RI uncertainty is $2.7 \% .{ }^{11}$ Second, the heterogeneous sample required different camera settings. Therefore, we have corrected detected scattering power for nonlinear camera gain response, which may change over time. Third, NTA determines the hydrodynamic diameter and therefore overestimates the physical diameter of a particle. Because $\sigma$ increases with diameter for particles $<200 \mathrm{~nm}$, an overestimation of the particle diameter causes an underestimation of the RI. Fourth, we have modeled vesicles as spheres with a uniform RI distribution. In practice, however, a vesicle consists of a low RI core enclosed by a several nm thick phospholipid shell with an RI of $1.46 \pm 0.06{ }^{56,57}$ With Mie theory, $\sigma$ of a shelled particle can be analytically described. Oscillations of $\sigma$ as a function of the diameter are likely to shift for a shelled particle compared to a solid particle, which warrants further investigation, since such a shift should be measurable with NTA.

In conclusion, we have demonstrated the first use of NTA to determine the RI of individual nanoparticles in suspension. A major advantage of this single particle RI determination over bulk RI measurements is that this method will give accurate RI measurements even if particles with different RI are present in the sample. Further, this method may be applied to differentiate between populations in a sample based on RI. We found that the mean RI of extracellular vesicles $<500 \mathrm{~nm}$ is 1.37 at $405 \mathrm{~nm}$. This hitherto unknown property of vesicles is essential to data interpretation and standardization of clinical research on vesicles. We expect that determination of the RI of nanoparticles with NTA will become an important tool in biomedical diagnostics, materials science, and oceanography, as well as other fields where the optical characterization of nanoparticles is of critical importance.

\section{ASSOCIATED CONTENT}

\section{S Supporting Information}

Transmission electron microscopy of urinary vesicles, light scattering calculations, camera settings, and particle tracking parameters. This material is available free of charge via the Internet at http://pubs.acs.org.

\section{AUTHOR INFORMATION}

\section{Corresponding Author}

*E-mail: e.vanderpol@amc.uva.nl. Address: Biomedical Engineering and Physics, Academic Medical Center, University of Amsterdam, Meibergdreef 9, Room: K01-226, P.O. Box 22660, 1100 DD, Amsterdam, The Netherlands. Phone: +31 (0) 20 5664386.

\section{Author Contributions}

E.v.d.P., R.N., and T.G.v.L. conceived and designed the research. E.v.d.P. acquired the data. E.v.d.P., F.A.C., R.N., and T.G.v.L. interpreted the data. The manuscript was written through contributions of all authors. All authors have given approval to the final version of the manuscript.

\section{Notes}

The authors declare no competing financial interest.

\section{ACKNOWLEDGMENTS}

The authors would like to acknowledge A. Grootemaat for excellent experimental support and P. Hole and A. Malloy from Nanosight, U.K., for valuable discussions. Part of this work is funded by the European Metrology Research Programme (EMRP) under the Joint Research Project HLT02 (www. metves.eu). The EMRP is jointly funded by the EMRP participating countries within the European Association of National Metrology Institutes and the European Union.

\section{ABBREVIATIONS}

NA, numerical aperture; NTA, nanoparticle tracking analysis; PBS, phosphate-buffered saline; PMMA, poly(methyl methacrylate); PS, polystyrene; PSD, particle size distribution; PTFE, polytetrafluoroethylene; RI, refractive index; RNA, 
ribonucleic acid; SD, standard deviation; Si, Silica; TEM, transmission electron microscopy

\section{REFERENCES}

(1) Knöner, G.; Parkin, S.; Nieminen, T. A.; Heckenberg, N. R.; Rubinsztein-Dunlop, H. Measurement of the index of refraction of single microparticles. Phys. Rev. Lett. 2006, 97 (15), 157402.

(2) Bohren, C. F.; Huffman, D. R. Absorption and scattering of light by small particles; Wiley: New York, 1983.

(3) Bendix, P. M.; Oddershede, L. B. Expanding the Optical Trapping Range of Lipid Vesicles to the Nanoscale. Nano Lett. 2011, 11 (12), 5431-5437.

(4) Righini, M.; Ghenuche, P.; Cherukulappurath, S.; Myroshnychenko, V.; de Abajo, F. J. G.; Quidant, R. Nano-Optical Trapping of Rayleigh Particles and Escherichia coli Bacteria with Resonant Optical Antennas. Nano Lett. 2009, 9 (10), 3387-3391.

(5) Charriere, F.; Marian, A.; Montfort, F.; Kuehn, J.; Colomb, T.; Cuche, E.; Marquet, P.; Depeursinge, C. Cell refractive index tomography by digital holographic microscopy. Opt. Lett. 2006, 31 (2), $178-180$

(6) Ferraris, C. F.; Guthrie, W.; Avilés, A. I.; Haupt, R.; MacDonald, B. S. Certification of SRM 114q: Part I; National Institute of Standards and Technology: Gaithersburg, MD, 2005; Volume 05, pp 260-261.

(7) Jewell, R. B.; Rathbone, R. F. Optical properties of coal combustion byproducts for particle-size analysis by laser diffraction. Coal Combust. Gasif. Prod. J. 2009, 1, 1-7.

(8) Mills, G. L.; Lane, P. A.; Weech, P. K. A guidebook to lipoprotein techniques; Elsevier: Amsterdam, 2000.

(9) Ungureanu, C.; Rayavarapu, R. G.; Manohar, S.; van Leeuwen, T. G. Discrete dipole approximation simulations of gold nanorod optical properties: Choice of input parameters and comparison with experiment. J. Appl. Phys. 2009, 105 (10), 102032.

(10) Forster, P.; Ramaswamy, V.; Artaxo, P.; Berntsen, T.; Betts, R.; Fahey, D.; Haywood, J.; Lean, J.; Lowe, D.; Myhre, G.; Nganga, J.; Prinn, R.; Raga, G.; Schultz, M.; Van Dorland, R. Changes in atmospheric constituents and in radiative forcing. In Climate change 2007: The physical science basis. Contribution of Working Group I to the Fourth Assessment Report of the Intergovernmental Panel on Climate Change, 20th ed.; Solomon, S., Qin, D., Manning, M., Chen, Z., Marquis, M., Averyt, K., Tignor, M., Miller, H., Eds.; Cambridge University Press: Cambridge, United Kingdom, 2007; pp 129-234.

(11) Miles, R. E. H.; Rudic, S.; Orr-Ewing, A. J.; Reid, J. P. Measurements of the wavelength dependent extinction of aerosols by cavity ring down spectroscopy. Phys. Chem. Chem. Phys. 2010, 12 (15), 3914-3920.

(12) Stramski, D. Refractive index of planktonic cells as a measure of cellular carbon and chlorophyll a content. Deep Sea Res., Part I 1999, 46 (2), 335-351.

(13) Mackowiak, S. A.; Schmidt, A.; Weiss, V.; Argyo, C.; von Schirnding, C.; Bein, T.; Brauchle, C. Targeted Drug Delivery in Cancer Cells with Red-Light Photoactivated Mesoporous Silica Nanoparticles. Nano Lett. 2013, 13 (6), 2576-2583.

(14) Jang, S. C.; Kim, O. Y.; Yoon, C. M.; Choi, D. S.; Roh, T. Y.; Park, J.; Nilsson, J.; Lotvall, J.; Kim, Y. K.; Gho, Y. S. Bioinspired Exosome-Mimetic Nanovesicles for Targeted Delivery of Chemotherapeutics to Malignant Tumors. ACS Nano 2013, 7 (9), 76987710.

(15) Gujrati, V.; Kim, S.; Kim, S. H.; Min, J. J.; Choy, H. E.; Kim, S. C.; Jon, S. Bioengineered Bacterial Outer Membrane Vesicles as CellSpecific Drug-Delivery Vehicles for Cancer Therapy. ACS Nano 2014, $8,1525-1537$.

(16) Ungureanu, C.; Kroes, R.; Petersen, W.; Groothuis, T. A. M.; Ungureanu, F.; Janssen, H.; van Leeuwen, F. W. B.; Kooyman, R. P. H.; Manohar, S.; van Leeuwen, T. G. Light Interactions with Gold Nanorods and Cells: Implications for Photothermal Nanotherapeutics. Nano Lett. 2011, 11 (5), 1887-1894.

(17) Manohar, S.; Ungureanu, C.; van Leeuwen, T. G. Gold nanorods as molecular contrast agents in photoacoustic imaging: the promises and the caveats. Contrast Media Mol. Imaging 2011, 6 (5), 389-400.

(18) Krpetic, Z.; Nativo, P.; See, V.; Prior, I. A.; Brust, M.; Volk, M. Inflicting Controlled Nonthermal Damage to Subcellular Structures by Laser-Activated Gold Nanoparticles. Nano Lett. 2010, 10 (11), 45494554.

(19) Rozhkova, E. A.; Ulasov, I.; Lai, B.; Dimitrijevic, N. M.; Lesniak, M. S.; Rajh, T. A High-Performance Nanobio Photocatalyst for Targeted Brain Cancer Therapy. Nano Lett. 2009, 9 (9), 3337-3342.

(20) Kumar, A.; Vemula, P. K.; Ajayan, P. M.; John, G. Silvernanoparticle-embedded antimicrobial paints based on vegetable oil. Nat. Mater. 2008, 7 (3), 236-241.

(21) Allen, N. S.; Edge, M.; Ortega, A.; Liauw, C. M.; Stratton, J.; McIntyre, R. B. Behaviour of nanoparticle (ultrafine) titanium dioxide pigments and stabilisers on the photooxidative stability of water based acrylic and isocyanate based acrylic coatings. Polym. Degrad. Stab. 2002, 78 (3), 467-478.

(22) Zhang, W.; Saliba, M.; Stranks, S. D.; Sun, Y.; Shi, X.; Wiesner, U.; Snaith, H. J. Enhancement of Perovskite-Based Solar Cells Employing Core-Shell Metal Nanoparticles. Nano Lett. 2013, 13 (9), $4505-4510$.

(23) Barer, R.; Tkaczyk, S. Refractive index of concentrated protein solutions. Nature 1954, 173, 821-822.

(24) Hart, S. J.; Leski, T. A. Refractive index determination of biological particles; ADA454180; U.S. Naval Research Laboratory: Washington, DC, Jul 31, 2006.

(25) Ma, X. Y.; Lu, J. Q.; Brock, R. S.; Jacobs, K. M.; Yang, P.; Hu, X. $\mathrm{H}$. Determination of complex refractive index of polystyrene microspheres from 370 to $1610 \mathrm{~nm}$. Phys. Med. Biol. 2003, 48 (24), $4165-4172$.

(26) Lang-Yona, M.; Rudich, Y.; Segre, E.; Dinar, E.; Abo-Riziq, A. Complex Refractive Indices of Aerosols Retrieved by Continuous Wave-Cavity Ring down Aerosol Spectrometer. Anal. Chem. 2009, 81 (5), 1762-1769.

(27) Varga, Z.; Yuana, Y.; Grootemaat, A. E.; van der Pol, E.; Gollwitzer, C.; Krumrey, M.; Nieuwland, R. Towards traceable size determination of extracellular vesicles. J. Extracell. Vesicles 2014, 3 (23298), 1-10.

(28) Barnes, M. D.; Lermer, N.; Whitten, W. B.; Ramsey, J. M. A CCD based approach to high-precision size and refractive index determination of levitated microdroplets using Fraunhofer diffraction. Rev. Sci. Instrum. 1997, 68 (6), 2287-2291.

(29) Chylek, P.; Ramaswamy, V.; Ashkin, A.; Dziedzic, J. M. Simultaneous determination of refractive-index and size of spherical dielectric particles from light-scattering data. Appl. Opt. 1983, 22 (15), 2302-2307.

(30) Pluchino, A. B.; Goldberg, S. S.; Dowling, J. M.; Randall, C. M. Refractive-index measurements of single micron-sized carbon particles. Appl. Opt. 1980, 19 (19), 3370-3372.

(31) Konokhova, A. I.; Yurkin, M. A.; Moskalensky, A. E.; Chernyshev, A. V.; Tsvetovskaya, G. A.; Chikova, E. D.; Maltsev, V. P. Light-scattering flow cytometry for identification and characterization of blood microparticles. J. Biomed. Opt. 2012, 17 (5), 0570061-0570068.

(32) Ackleson, S. G.; Spinrad, R. W. Size and refractive-index of individual marine particulates - a flow cytometric approach. Appl. Opt. 1988, 27 (7), 1270-1277.

(33) Kolesnikova, I. V.; Potapov, S. V.; Yurkin, M. A.; Hoekstra, A. G.; Maltsev, V. P.; Semyanov, K. A. Determination of volume, shape and refractive index of individual blood platelets. J. Quant. Spectrosc. Radiat. Transfer 2006, 102 (1), 37-45.

(34) Doornbos, R. M. P.; Schaeffer, M.; Hoekstra, A. G.; Sloot, P. M. A.; deGrooth, B. G.; Greve, J. Elastic light-scattering measurements of single biological cells in an optical trap. Appl. Opt. 1996, 35 (4), 729734.

(35) Ulanowski, Z.; Greenaway, R. S.; Kaye, P. H.; Ludlow, I. K. Laser diffractometer for single-particle scattering measurements. Meas. Sci. Technol. 2002, 13 (3), 292-296. 
(36) van der Pol, E.; Böing, A. N.; Harrison, P.; Sturk, A.; Nieuwland, R. Classification, functions and clinical relevance of extracellular vesicles. Pharmacol Rev. 2012, 64 (3), 676-705.

(37) Dragovic, R. A.; Gardiner, C.; Brooks, A. S.; Tannetta, D. S.; Ferguson, D. J. P.; Hole, P.; Carr, B.; Redman, C. W. G.; Harris, A. L.; Dobson, P. J.; Harrison, P.; Sargent, I. L. Sizing and phenotyping of cellulars vesicles using Nanoparticle Tracking Analysis. Nanomedicine 2011, 7 (6), 780-788.

(38) Braeckmans, K.; Buyens, K.; Bouquet, W.; Vervaet, C.; Joye, P.; De Vos, F.; Plawinski, L.; Doeuvre, L.; Angles-Cano, E.; Sanders, N. N.; Demeester, J.; De Smedt, S. C. Sizing Nanomatter in Biological Fluids by Fluorescence Single Particle Tracking. Nano Lett. 2010, 10 (11), 4435-4442.

(39) van der Pol, E.; Coumans, F.; Grootemaat, A. E.; Gardiner, C.; Sargent, I. L.; Harrison, P.; Sturk, A.; van Leeuwen, T. G.; Nieuwland, R. Particle size distribution of exosomes and microvesicles by transmission electron microscopy, flow cytometry, nanoparticle tracking analysis, and resistive pulse sensing. J. Thromb. Haemostasis 2014, 12 (7), 1182-1192.

(40) Biller, S. J.; Schubotz, F.; Roggensack, S. E.; Thompson, A. W.; Summons, R. E.; Chisholm, S. W. Bacterial vesicles in marine ecosystems. Science 2014, 343 (6167), 183-186.

(41) van der Pol, E.; Hoekstra, A. G.; Sturk, A.; Otto, C.; van Leeuwen, T. G.; Nieuwland, R. Optical and non-optical methods for detection and characterization of microparticles and exosomes. J. Thromb. Haemostasis 2010, 8 (12), 2596-2607.

(42) McMeekin, T. L.; Wilensky, M.; Groves, M. L. Refractive indices of proteins in relation to amino acid composition and specific volume. Biochem. Biophys. Res. Commun. 1962, 7 (2), 151-156.

(43) Shih, S. J.; Yagami, M.; Tseng, W. J.; Lin, A. Validation of a quantitative method for detection of adenovirus aggregation. Bioprocess. J. 2011, 9 (2), 25-33.

(44) Oster, G. Two-phase formation in solutions of tobacco mosaic virus and the problem of long-range forces. J. Gen. Physiol. 1950, 33 (5), 445-473.

(45) van der Pol, E.; van Gemert, M. J. C.; Sturk, A.; Nieuwland, R.; van Leeuwen, T. G. Single versus swarm detection of microparticles and exosomes by flow cytometry. J. Thromb. Haemostasis 2012, 10 (5), 919-30.

(46) Tatischeff, I.; Larquet, E.; Falcón-Pérez, J. M.; Turpin, P. Y.; Kruglik, S. G. Fast characterisation of cell-derived extracellular vesicles by nanoparticles tracking analysis, cryo-electron microscopy, and Raman tweezers microspectroscopy. J. Extracell. Vesicles 2012, 1, 1-11.

(47) Gardiner, C.; Ferreira, Y. J.; Dragovic, R. A.; Redman, C. W. G.; Sargent, I. L. Extracellular vesicle sizing and enumeration by nanoparticle tracking analysis. J. Extracell. Vesicles 2013, 2 (19671), $1-11$.

(48) Mätzler, C. MATLAB functions for Mie scattering and absorption, 2002-11 ed.; Institut für Angewandte Physik: Hamburg, 2002; p 02.

(49) Kasarova, S. N.; Sultanova, N. G.; Ivanov, C. D.; Nikolov, I. D. Analysis of the dispersion of optical plastic materials. Opt. Mater. 2007, 29 (11), 1481-1490.

(50) The Matlab particle tracking code repository; Georgetown University: Washington, DC, 2008.

(51) Hart, S. J.; Terray, A. V. Refractive-index-driven separation of colloidal polymer particles using optical chromatography. Appl. Phys. Lett. 2003, 83 (25), 5316-5318.

(52) Michalet, X.; Berglund, A. J. Optimal diffusion coefficient estimation in single-particle tracking. Phys. Rev. E 2012, 85 (6).

(53) Cachau R. E.; Braden B. C.; Collins J. R.; Casas-Finet J. R. Nanoparticles characterization using HDR-NTA image analysis; SPIE Photonics West, San Francisco, CA, USA, February 2014; pp 895434.

(54) Kindt, J. D. Optofluidic intracavity spectroscopy for spatially, temperature, and wavelength dependent refractometry. M.Sc. Thesis, Colorado State University, Fort Collins, CO, 2012.

(55) Chandler, W. L.; Yeung, W.; Tait, J. F. A new microparticle size calibration standard for use in measuring smaller microparticles using a new flow cytometer. J. Thromb. Haemostasis 2011, 9, 1216-1224.
(56) van Manen, H. J.; Verkuijlen, P.; Wittendorp, P.; Subramaniam, V.; van den Berg, T. K.; Roos, D.; Otto, C. Refractive index sensing of green fluorescent proteins in living cells using fluorescence lifetime imaging microscopy. Biophys. J. 2008, 94 (8), L67-L69.

(57) Beuthan, J.; Minet, O.; Helfmann, J.; Herrig, M.; Muller, G. The spatial variation of the refractive index in biological cells. Phys. Med. Biol. 1996, 41 (3), 369-382.

(58) Foladori, P.; Quaranta, A.; Ziglio, G. Use of silica microspheres having refractive index similar to bacteria for conversion of flow cytometric forward light scatter into biovolume. Water Res. 2008, 42 (14), 3757-3766.

(59) Barer, R. Refractometry and interferometry of living cells. J. Opt. Soc. Am. 1957, 47 (6), 545-556. 